\title{
Identification and Expression Profiling of the BTB Domain-Containing Protein Gene Family in the Silkworm, Bombyx mori
}

\author{
Daojun Cheng, Wenliang Qian, Meng Meng, Yonghu Wang, Jian Peng, and Qingyou Xia
}

State Key Laboratory of Silkworm Genome Biology, Southwest University, No. 2 Tiansheng Street, Beibei District,
Chongqing 400715, China

Correspondence should be addressed to Qingyou Xia; xiaqy@swu.edu.cn

Received 24 January 2014; Revised 5 April 2014; Accepted 7 April 2014; Published 6 May 2014

Academic Editor: Margarita Hadzopoulou-Cladaras

Copyright (C) 2014 Daojun Cheng et al. This is an open access article distributed under the Creative Commons Attribution License, which permits unrestricted use, distribution, and reproduction in any medium, provided the original work is properly cited.

\begin{abstract}
The BTB domain is a conserved protein-protein interaction motif. In this study, we identified 56 BTB domain-containing protein genes in the silkworm, in addition to 46 in the honey bee, 55 in the red flour beetle, and 53 in the monarch butterfly. Silkworm BTB protein genes were classified into nine subfamilies according to their domain architecture, and most of them could be mapped on the different chromosomes. Phylogenetic analysis suggests that silkworm BTB protein genes may have undergone a duplication event in three subfamilies: BTB-BACK-Kelch, BTB-BACK-PHR, and BTB-FLYWCH. Comparative analysis demonstrated that the orthologs of each of $13 \mathrm{BTB}$ protein genes present a rigorous orthologous relationship in the silkworm and other surveyed insects, indicating conserved functions of these genes during insect evolution. Furthermore, several silkworm BTB protein genes exhibited sex-specific expression in larval tissues or at different stages during metamorphosis. These findings not only contribute to a better understanding of the evolution of insect BTB protein gene families but also provide a basis for further investigation of the functions of BTB protein genes in the silkworm.
\end{abstract}

\section{Introduction}

The BTB (bric-a-brac, tramtrack, broad complex) domain, also known as the POZ domain, is an evolutionarily conserved protein-protein interaction motif consisting of approximately 100 amino acid residues. This domain was originally identified in the bric-a-brac, tramtrack, and broad complex proteins in the fruit fly (Drosophila melanogaster) [1]. The BTB domain-containing protein (referred to as "BTB protein" hereafter) gene family is characterized by the presence of one or more BTB domains in each family member and is found widely in eukaryotes [1-6]. Several other types of functional domains, including zinc finger (ZF), Kelch, BTB and C-terminal Kelch (BACK), meprin and TRAF homology (MATH), ankyrin repeats (ANK), PHR, and Ras homology (Rho) domains, are also found in some BTB proteins. According to the presence of these domains, the BTB protein gene family can be divided into multiple subfamilies, including BTB only proteins, BTB-ZF proteins, BTB-Kelch proteins, BTB-BACK proteins, BTB-BACK-Kelch proteins, MATH-BTB proteins, BTB-ANK proteins, BTBBACK-PHR proteins, and Rho-BTB proteins $[3,5,6]$.

The members of the BTB protein gene family are functionally involved in a variety of biological events in eukaryotes, including developmental timing [7], oogenesis [8], organ formation [9], cell development [10, 11], cell apoptosis $[12,13]$, protein degradation [14-17], cytokinesis $[18,19]$, and tumorigenesis $[20,21]$. Furthermore, the functions of BTB proteins are mainly governed by two prominent mechanisms [5]: BTB domain-based protein-protein interactions [22-24] and transcriptional regulation by DNA binding domains such as zinc finger domains [6, 25-28].

Genome-wide characterization of the BTB protein gene family has been performed in several species. The numbers of genes belonging to this family vary greatly among species [6]; 183 are found in humans (Homo sapiens), 178 
in the nematode (Caenorhabditis elegans), 195 in the mouse (Mus musculus), 77 in the mouse-ear cress (Arabidopsis thaliana), 85 in the fruit fly, and 85 in the malaria mosquito (Anopheles gambiae). The silkworm (Bombyx mori) is considered an economically important insect and has been used widely for determining the genetic basis of biological events in the order Lepidoptera. To date, only one BTB protein, broad complex (BR-C) protein of the BTB-ZF subfamily, has been identified in the silkworm, where it has been shown to mediate ecdysone signaling $[29,30]$. In the present study, we systematically identified BTB protein genes in the silkworm using the silkworm genome sequence [31], compared them with the BTB protein genes of other insects, and profiled their spatial and temporal expression patterns.

\section{Materials and Methods}

2.1. Genome-Wide Identification of BTB Protein Genes. BTB protein genes were identified in thesilkworm genome using the amino acid sequence of the conserved BTB domain as a query against the gene collections downloaded from two silkworm genome databases, SilkDB (http://www.silkdb.org/silkdb/) and KAIKObase (http://sgp .dna.affrc.go.jp/KAIKObase/), in a local BLASTp search. The predicted BTB protein genes were further annotated through an online BLAST search in NCBI. The same approach was used to identify BTB protein genes in four other insects: the fruit fly, the honey bee (Apis mellifera, Hymenoptera), the red flour beetle (Tribolium castaneum, Coleoptera), and the monarch butterfly (Danaus plexippus, Lepidoptera). Two online programs, SMART (http://smart.embl-heidelberg.de/) [32] and a CDD search program (http://www.ncbi .nlm.nih.gov/Structure/cdd/wrpsb.cgi) [33], were used to characterize the domain architecture of the predicted BTB protein genes in the silkworm genome. According to the gene-naming principles established by the Gene Nomenclature Committee of the Human Genome Organization (HGNC), "BTB" plus the initial word in the descriptions of other domains was used for the abbreviated names of the BTB protein genes identified in this study.

\subsection{Chromosomal Distribution and Subcellular Localization.} Silkworm BTB protein genes were mapped on the chromosomes based on the single- nucleotide polymorphism (SNP) linkage map for the silkworm [34]. The subcellular locations and other characteristics of silkworm BTB proteins were predicted by two programs: WoLF PSORT (http://wolfpsort.org) [35] and TargetP (http://www.cbs.dtu.dk/services/TargetP).

2.3. Construction of Phylogenetic Trees. The full amino acid sequences and BTB domain sequences of the identified BTB proteins of the silkworm and other surveyed insects were used to perform a multiple sequence alignment by the ClustalX program [36]. The alignment result was further used to build a neighbor-joining phylogenetic tree with a bootstrap of 1,000 replicates. The phylogenetic tree was refined by the MEGA4 program [37].
2.4. Microarray Analysis of Spatiotemporal Gene Expression. Based on microarray data for gene expression in various tissues of silkwormlarvae on day 3 of the fifth instar (L5D3) [38], we determined the expression patterns of silkworm BTB protein genes on L5D3 in nine larval tissues, including ovary, testis, head, integument, fat body, midgut, hemocyte, Malpighian tubule, anterior/middle silk gland (A/MSG), and posterior silk gland (PSG). The approach used in this analysis was identical to that described in a previous report [38]. In addition, based on the microarray data for gene expression during silkworm metamorphosis, which involves the transition from late larva to pupa and then to adult (unpublished), we analyzed the expression patterns of silkworm BTB protein genes at 19 developmental time points. These time points included day 4 of the fifth larval instar: (L5D4), L5D5, L5D6, L5D7, beginning of wandering for spinning (W0), 12 hours after wandering (W12h), W24h, W36h, W48 h (completing spinning), beginning of pupation (P0), day 1 following pupation (P1), P2, P3, P4, P5, P6, P7, P8, and adult. Gene expression in the silkworm individuals on L5D3 was used as a control. Microarray hybridization and raw data processing were performed as previously described [38]. If the signal intensity of the expression of a BTB protein gene exceeded 400 units at a time point, this gene was considered to be expressed at that time point. We then calculated the ratio of the expression level of each $\mathrm{BTB}$ protein gene inthe silkworm individuals at each time point to that in the L5D3 control. These ratios were further used to examine dynamic changes in the expression levels of BTB protein genes during silkworm metamorphosis using the GeneCluster 2.0 program [39].

\subsection{RT-PCR Examination of Spatiotemporal Gene Expres-} sion. RT-PCR experiments were further used to examine the expression patterns of silkworm BTB protein genes in multiple tissues of silkworm larvae on L5D3. Silkworms of the Dazao strain were reared on fresh mulberry leaves at $25^{\circ} \mathrm{C}$ under 12-hour light/12-hour dark cycle. Silkworm larval tissues, including silk gland, midgut, fat body, hemocyte, head, integument, Malpighian tubule, ovary, and testis, were isolated from female and male larval individuals on L5D3. The procedures for RNA extraction, cDNA synthesis, and RTPCR reaction were the same as previously described [40]. The primers used in these experiments are listed in Table S1 (see Table S1 in Supplementary Material available online at http://dx.doi.org/10.1155/2014/865065).

\section{Results}

3.1. Identification of BTB Protein Genes in the Silkworm Genome. To identify BTB protein genes in the silkworm genome, we used the amino acid sequence of a common BTB domain (SMART accession number: SM00225) and the BTB domain sequence of BR-C protein as queries to search against the predicted silkworm gene collection. As a result, 56 BTB protein genes were retrieved from the silkworm genome (Table 1). SMART and CDD search-based domain analysis showed that, in addition to the BTB domain, most 


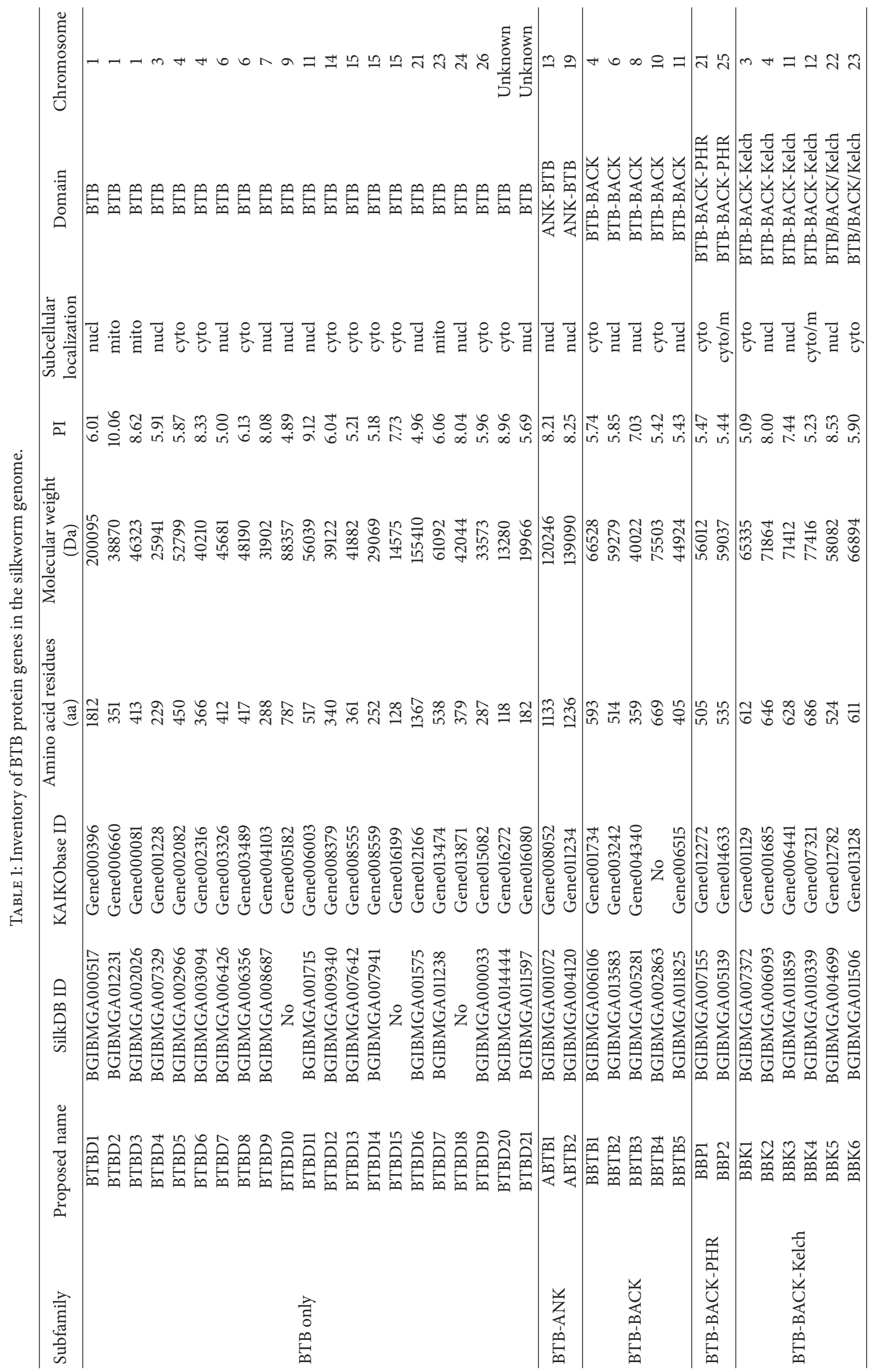




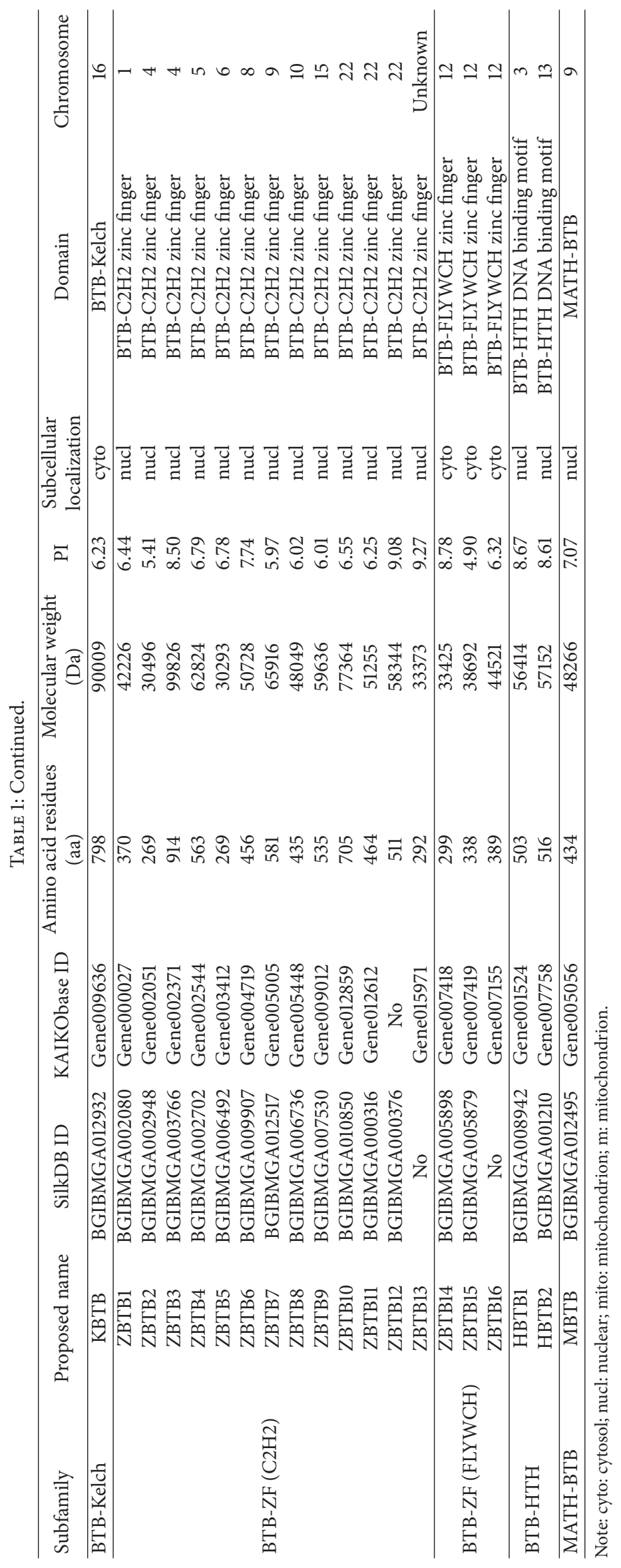




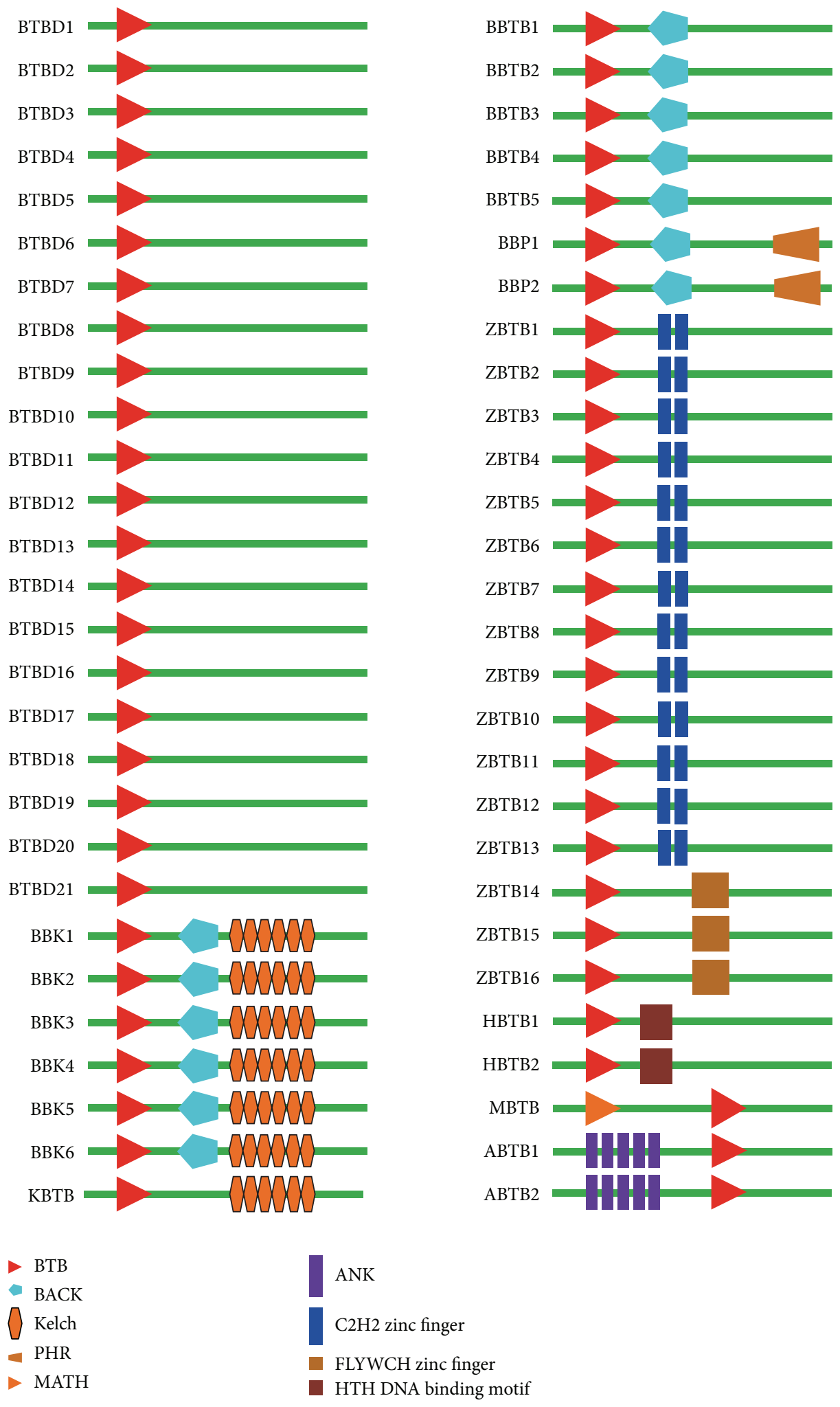

FIGURE 1: Architecture of functional domains in silkworm BTB protein genes. The conserved BTB domain and other types of functional domains are marked with different signs and colors as indicated below the schematic architecture. Domain names refer to the resource from the SMART database. 

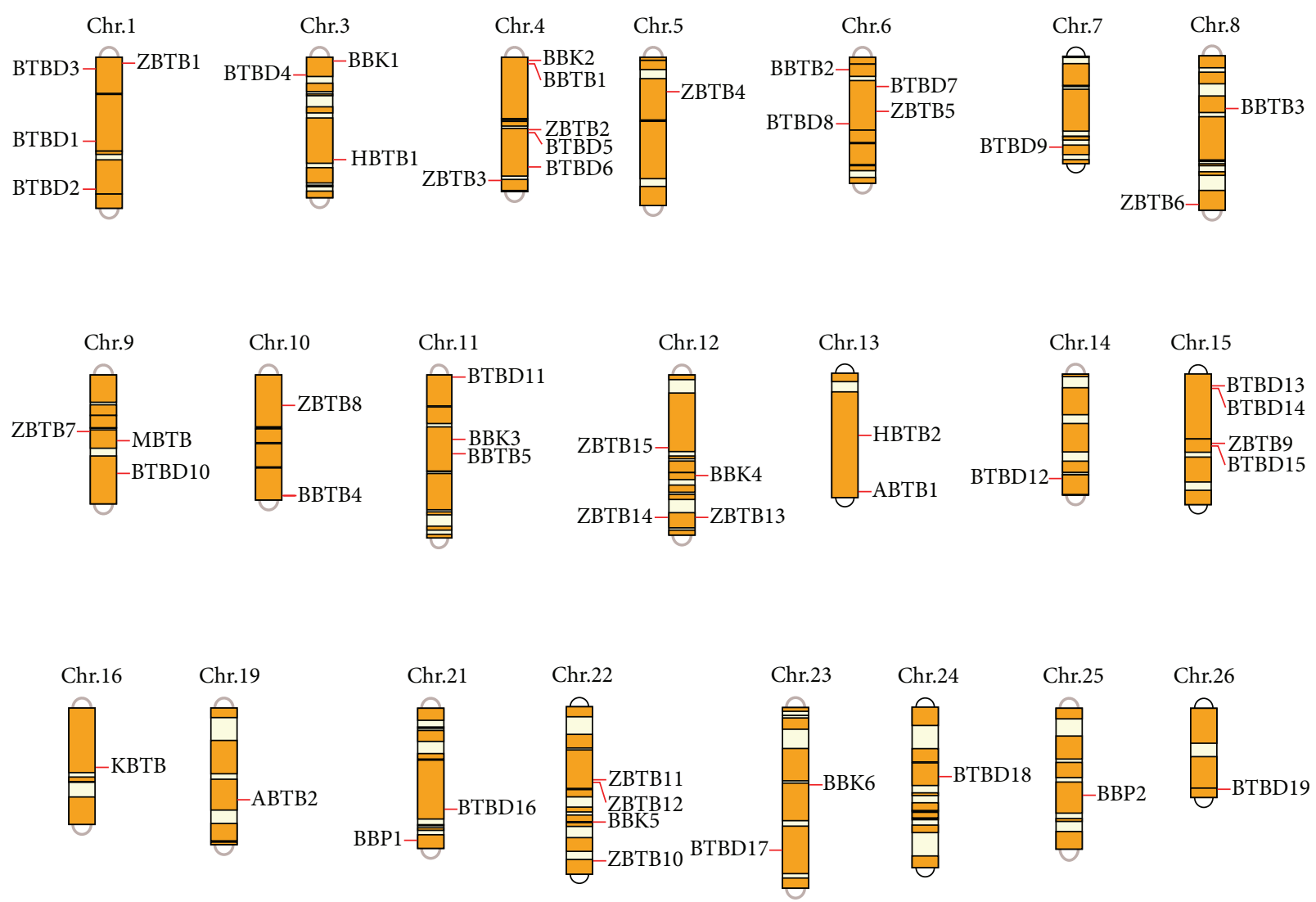

FIGURE 2: Chromosomal distribution of silkworm BTB protein genes. In total, 53 of the identified silkworm BTB protein genes (i.e., all except BTBD20, BTBD21, and ZBTB13) were localized to specific chromosomes based on the whole-genome sequence and single-nucleotide polymorphism (SNP) marker linkage map for the silkworm. The chromosome number of each gene was indicated above.

silkworm BTB protein genes also contained one or more other functional domain types such as ANK, BACK, PHR, Kelch, zinc finger, HTH DNA binding motif, and MATH domains (Table 1 and Figure 1).

According to previous reports [6] and on the basis of the presence of other types of domain, we classified the identified silkworm BTB protein genes into nine subfamilies: BTB only (BTBD), BTB-ANK (ABTB), BTB-BACK (BBTB), BTB-BACK-PHR (BBP), BTB-BACK-Kelch (BBK), BTBKelch (KBTB), BTB-ZF (ZBTB including those with $\mathrm{C} 2 \mathrm{H} 2$ or FLYWCH zinc finger domains), BTB-HTH (HBTB), and MATH-BTB (MBTB) subfamilies. The silkworm BTB protein gene subfamilies were named in an abbreviated format based on the principles for the nomenclature of BTB proteins described by the HUGO Gene Nomenclature Committee (HGNC) and on previous reports [6]. These nine subfamilies contain $22,2,5,2,6,1,15,2$, and 1 members, respectively (Table 1 and Figure 1).

\subsection{Chromosomal Distribution and Subcellular Location of} Silkworm BTB Protein Genes. Based on the linkage map [34] and the updated genome assembly for the silkworm [31], 53 of the 56 identified silkworm BTB protein genes (excluding BTBD20, BTBD21, and ZBTB13) were assigned to 22 chromosomes (Table 1 and Figure 2). The members of each
BTB protein subfamily were distributed dispersedly on the different chromosomes.

We predicted the subcellular location of silkworm BTB proteins using the online programs WoLF PROST and TargetP. As expected, BTB proteins containing $\mathrm{C} 2 \mathrm{H} 2$ zinc finger or HTH DNA binding motifs, two domains associated with activation of the transcription of target genes, were predicted to be located within the nucleus (Table 1); the BTBANK and MATH-BTB subfamilies were also classified among the nuclear proteins. However, BTB proteins containing the FLYWCH zinc finger domain and the members of the BTB-BACK-PHR subfamily were predicted to be located in the cytosol. The members of each of the remaining BTB protein subfamilies were assigned to the nucleus, cytosol, or mitochondrion.

3.3. Phylogenetic Tree of Silkworm BTB Protein Genes. Given that BTB fold of the BTB proteins has been shown to be structurally well conserved [6], we used the amino acid sequences of BTB domains from the identified silkworm BTB proteins to generate a phylogenetic tree of silkworm BTB protein genes. As shown in Figure 3(a), the members of several BTB protein gene subfamilies, including BTB-BACK-Kelch, BTB-BACKPHR, and BTB-FLYWCH proteins (ZBTB14, ZBTB14, and $Z B T B 15)$ of the BTB zinc finger subfamily, were grouped more 


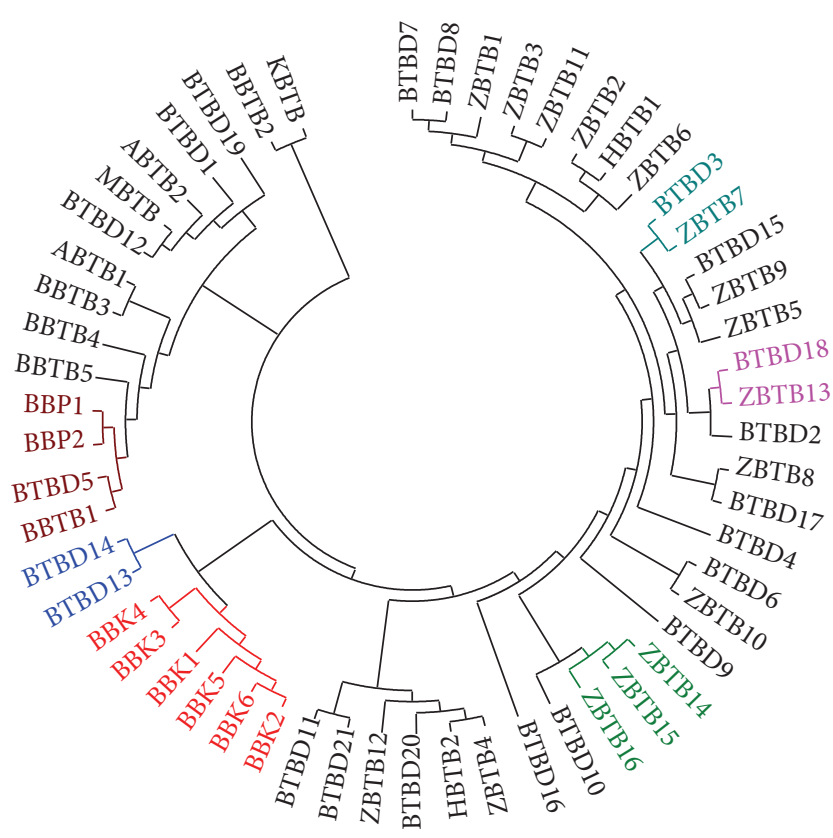

(a)

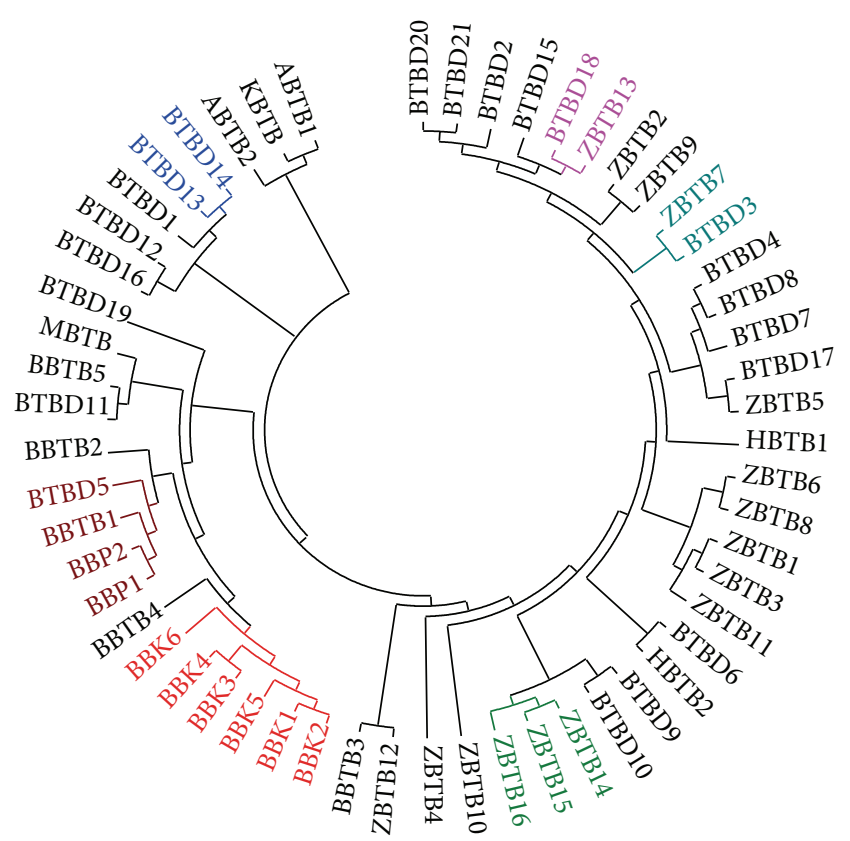

(b)

FIGURE 3: Phylogenetic tree of silkworm BTB protein genes. A neighbor-joining phylogenetic tree of the identified silkworm BTB protein genes was constructed using the ClustalX program and visualized using the MEGA4 software. (a) BTB domain sequence-based phylogenetic tree. (b) Complete amino acid sequence-based phylogenetic tree. Similar grouping clades between these two phylogenetic trees are indicated by the same colors.

closely than other BTB protein genes. Curiously, the members of each of the remaining subfamilies, except for BTB-Kelch and MATH-BTB, each of which contained single gene, were grouped irregularly with the members of other subfamilies.

We also constructed a phylogenetic tree of silkworm BTB protein genes using the complete amino acid sequences predicted by these genes. As shown in Figure 3(b), the members of three subfamilies, BTB-BACK-Kelch, BTB-BACK-PHR, and BTB-FLYWCH, were tightly grouped together, which is consistent with the observation from the BTB domain sequence-based phylogenetic tree. This indicates that the BTB protein genes in these three subfamilies have experienced a duplication event and may have similar biological functions. Moreover, several grouping clades were also similar to those indicated by the BTB domain-based phylogenetic tree, including the clade of $B T B D 13$ and $B T B D 14$, the clade of BTBD3 and ZBTB3, the clade of BTBD18 and ZBTB13, and the clade including $B T B D 5, B B T B 1$, and the BTB-BACK-PHR subfamily. The remaining BTB protein genes were grouped into different clades in an irregular manner.

3.4. Comparison of the BTB Protein Genes of the Silkworm with Those of Other Insects. To comprehensively characterize the evolution of insect BTB protein genes, we identified BTB protein gene families in four insect species for which the whole-genome sequence is available. As listed in Table 2 and Table S2, 44 BTB protein genes were found in the fruit fly, 46 in the honey bee, 55 in the red flour beetle, and 53 in the monarch butterfly. Intriguingly, the BTB-ANK and MATH-BTB subfamilies contained the same numbers of genes, two and one, respectively, in the silkworm and in the other four surveyed insect species. Furthermore, the BTB-Kelch subfamily was found in only three of the four additional surveyed insect species (not in the honey bee). The BTB-BACK-PHR subfamily was found only in the silkworm, honey bee, and monarch butterfly, whereas the RhoBTB subfamily was present in the fruit fly, red flour beetle, and monarch butterfly.

We constructed a phylogenetic tree including all of the BTB protein genes identified in the silkworm and other surveyed insects using the amino acid sequences of their BTB domains (Figure 4). In this phylogenetic tree, the members of two subfamilies, MATH-BTB and BTB-BACKKelch, were always grouped together. Furthermore, although most members of the BTB-ZF subfamily were promiscuously grouped into different clades with members of other BTB subfamilies, BmZBTB6 and BmZBTB5, two silkworm BTB$\mathrm{ZF}$ protein genes that encode $\mathrm{BR}-\mathrm{C}$ and fruitless, respectively, were grouped together into a clade with their homologs from other surveyed insects and they showed an orthologous $1: 1: 1: 1: 1$ relationship among all five insect species. This was the same as grouping clades containing the orthologs of the following BTB protein genes from the silkworm: $B m Z B T B 3$, BmZBTB7, BmZBTB9, BmZBTB10, BmBTBD1, BmBTBD15, $B m A B T B 1$, and BmBBTB4.

3.5. Tissue Expression Profile of Silkworm BTB Protein Genes. A previous study reported a microarray analysis of genomewide gene expression in multiple tissues of silkworm larvae on day 3 of the fifth instar (L5D3) [38]. Based on 


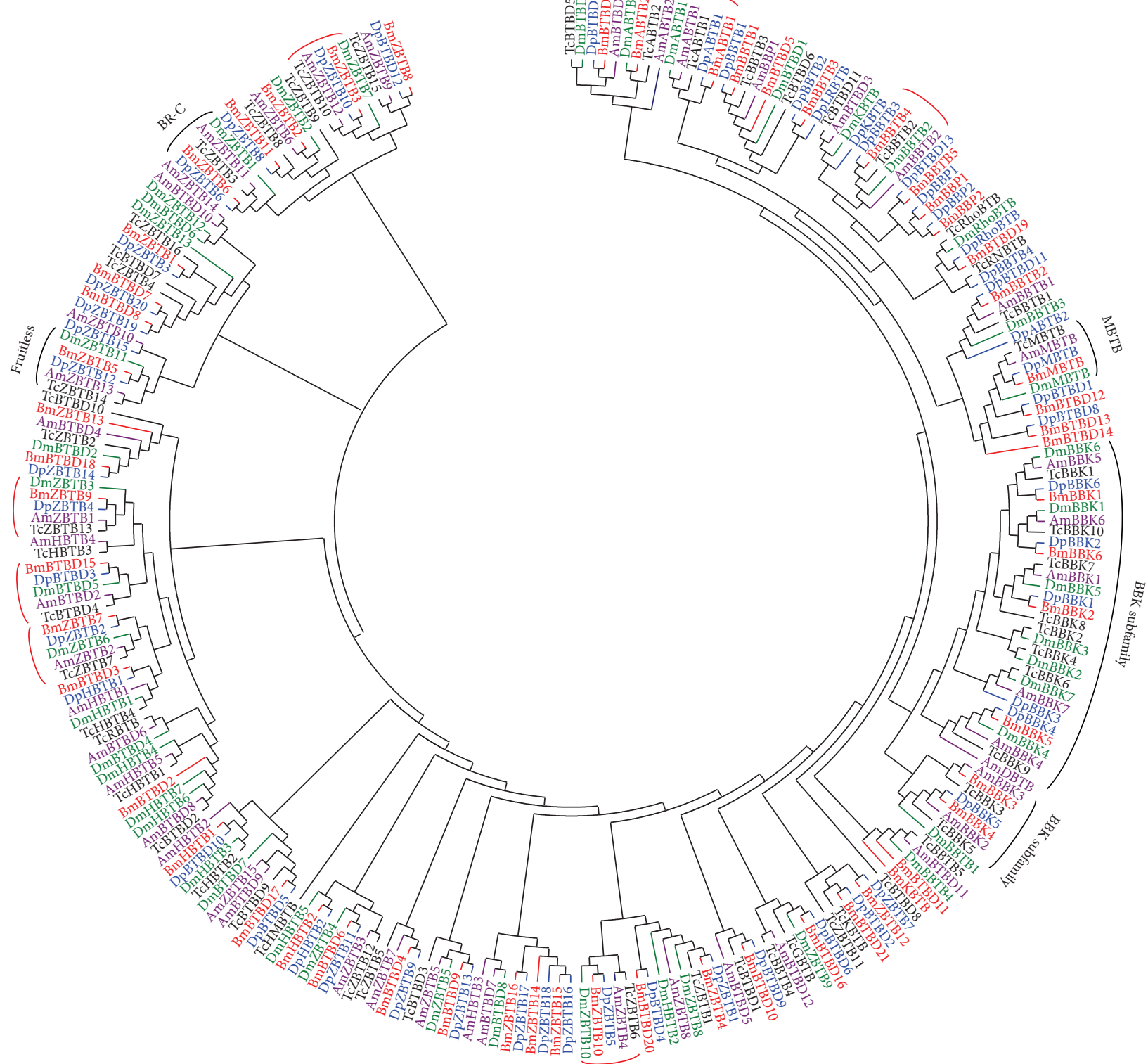

\footnotetext{
$\mathrm{Bm}=$ Bombyx mori

$\mathrm{Dr}=$ Drosophila melanogaster

$\mathrm{Dp}=$ Danaus plexippus
}

Am $=$ Apis mellifera

$\mathrm{Tc}=$ Tribolium castaneum

FIgURE 4: Phylogenetic relationships of the BTB protein genes of the silkworm with those of other surveyed insects. Based on the amino acid sequences of the BTB domains, a neighbor-joining phylogenetic tree of all insect BTB protein genes was constructed using the ClustalX program and visualized using the MEGA4 software. The grouping clade for the orthologs of a silkworm BTB protein gene that showed a $1: 1: 1: 1: 1$ orthologous relationship among all five surveyed insect species is indicated by a red cambered line. The names of all BTB protein genes from a single insect species are indicated in the same color.

microarray data from that study, we analyzed the expression patterns of silkworm BTB protein genes in larval tissues and found that 25 of the identified silkworm BTB protein genes were expressed in at least one larval tissue (Figure 5). Notably, 22 BTB protein genes were highly expressed in the gonads of the silkworm, and most of these genes showed higher expression in the testis than in the ovary. Interestingly, $A B T B 2, B B K 3$, and $B B K 4$ were specifically expressed in the testis. ZBTB6 expression was present in the Malpighian tubule, integument, fat body, and midgut. ZBTB4 was mainly expressed in the hemocytes, head, and integument. MBTB showed ubiquitous expression in the ovary, testis, midgut, and Malpighian tubule. In addition, RT-PCR examination confirmed the testis-specific expression of $A B T B 2, B B K 3$, and $B B K 4$ in silkworm larvae (Figure 6). 


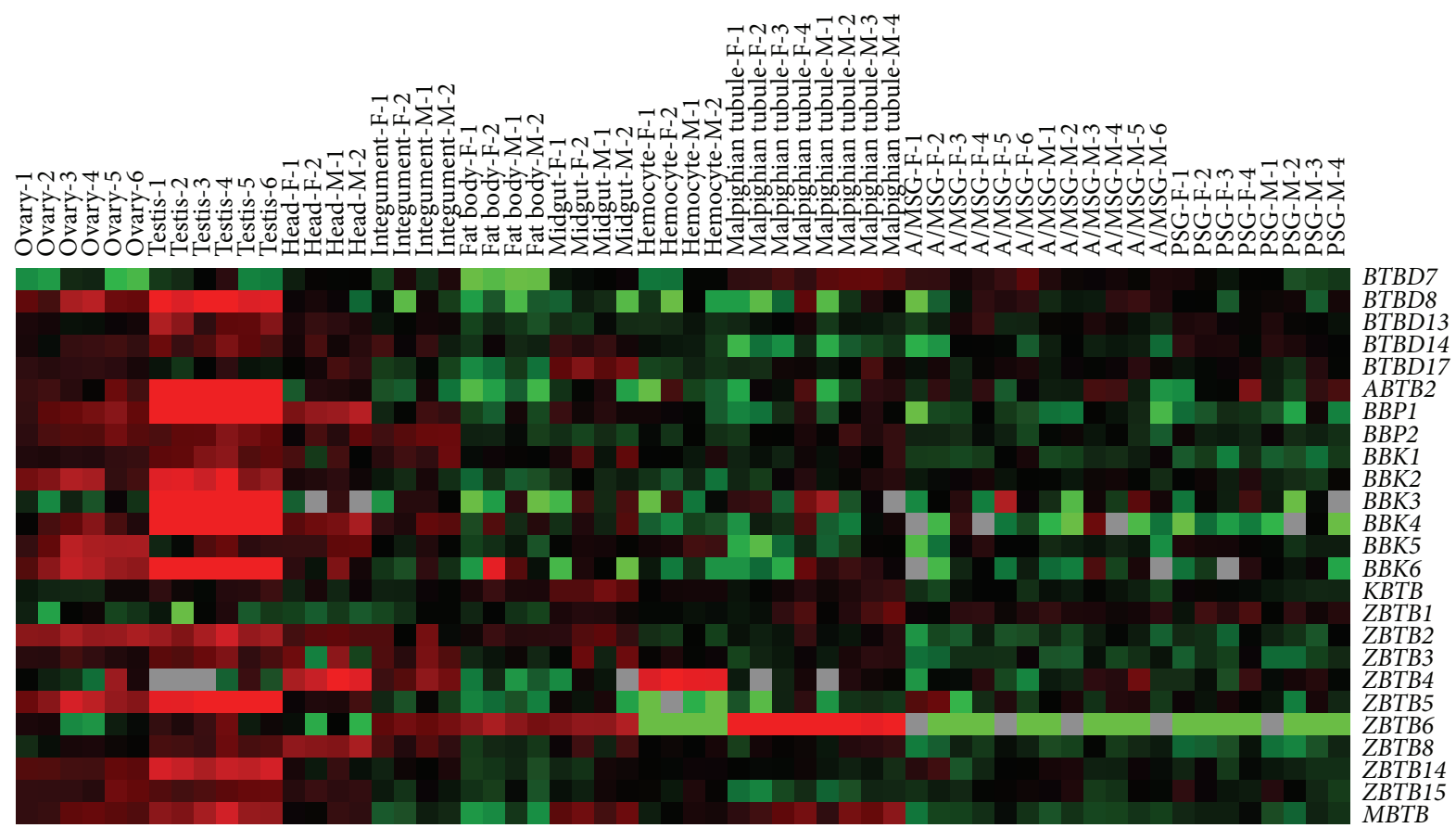

m†イーnm

FIGURE 5: Microarray-based expression profiling of BTB protein genes in multiple tissues of silkworm larvae. Microarray data representing genome-wide gene expression in multiple tissues of silkworm larvae on day 3 of the fifth instar were downloaded from the SilkDB database. Each tissue sample was analyzed using at least two biological repeats, which are indicated with different Arabic numerals. F: female; M: male; A/MSG: anterior/median silk gland; PSG: posterior silk gland.

TABLE 2: Number variation of the members of each BTB protein subfamily in the silkworm and other insects.

\begin{tabular}{|c|c|c|c|c|c|}
\hline Subfamily & $\begin{array}{c}\text { Silkworm } \\
\text { (Lepidoptera) }\end{array}$ & $\begin{array}{l}\text { Fruit fly } \\
\text { (Diptera) }\end{array}$ & $\begin{array}{c}\text { Honey bee } \\
\text { (Hymenoptera) }\end{array}$ & $\begin{array}{l}\text { Red flour beetle } \\
\text { (Coleoptera) }\end{array}$ & $\begin{array}{c}\text { Monarch butterfly } \\
\text { (Lepidoptera) }\end{array}$ \\
\hline BTB only & 22 & 8 & 12 & 11 & 13 \\
\hline BTB-ANK & 2 & 2 & 2 & 2 & 2 \\
\hline BTB-BACK & 5 & 4 & 2 & 5 & 4 \\
\hline BTB-BACK-PHR & 2 & - & 1 & - & 2 \\
\hline BTB-BACK-Kelch & 6 & 7 & 7 & 10 & 6 \\
\hline BTB-Kelch & 1 & 1 & - & 1 & 1 \\
\hline BTB-ZF (C2H2) & 12 & 11 & 14 & 15 & 15 \\
\hline BTB-ZF (FLYWCH) & 3 & 2 & 1 & 1 & 5 \\
\hline BTB-HTH & 2 & 7 & 5 & 4 & 2 \\
\hline МATH-BTB & 1 & 1 & 1 & 1 & 1 \\
\hline RhoBTB & - & 1 & - & 1 & 1 \\
\hline Others & - & - & 1 & 4 & 1 \\
\hline Total & 56 & 44 & 46 & 55 & 53 \\
\hline
\end{tabular}

Note: - represents no identification.

3.6. Developmental Expression Profile of the Silkworm BTB Protein Genes. Using the microarray data on gene expression during silkworm metamorphosis from the larval to adult stages, we surveyed the developmental expression profile of the silkworm BTB protein genes. The results showed that 27 of the identified BTB protein genes were expressed during silkworm metamorphosis (Figure 7). Remarkably, most of these BTB protein genes showed a high expression during the pupa-adult transition. Moreover, the expression of eight silkworm BTB protein genes, including BTBD6, $A B T B 2$, $B B T B 3, B B K 3, B B K 4, B B K 5, Z B T B 5$, and ZBTB7, displayed sexual dimorphism; the expression of $B T B D 6, A B T B 2, B B K 3$, 


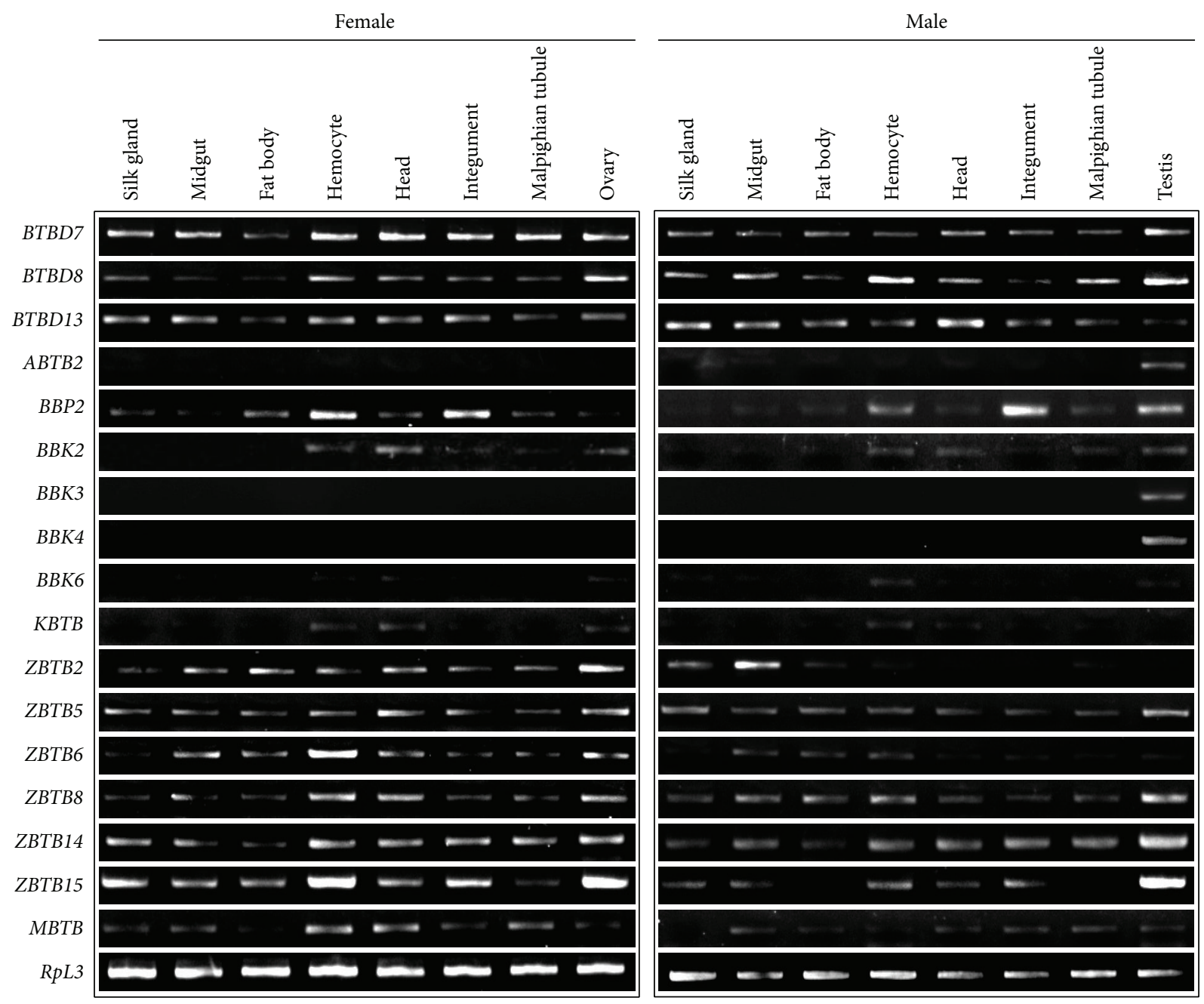

FIGURE 6: RT-PCR examination of expression profiles of the BTB protein genes in silkworm larval tissues. The expression profiles of the BTB protein genes in multiple tissues of silkworm larvae on day 3 of the fifth instar (L5D3) were further examined in RT-PCR experiments. The silkworm $R p L 3$ gene was used as a control.

and $B B K 4$ was male-specific, whereas that of $B B T B 3, B B K 5$, $Z B T B 5$, and ZBTB7 was female-specific.

\section{Discussion}

Members of the BTB protein gene family are characterized by the presence of a BTB domain that mediates proteinprotein interactions $[22,41,42]$. In this study, 56 BTB protein genes were identified in the silkworm genome. Similar to the findings of a previous study [6], in addition to the typical BTB domain, most of the identified silkworm BTB protein genes also contained other types of functional domains such as zinc finger, BACK, Kelch, ANK, MATH, PHR, and HTH domains. Based on the presence of the other domains, we classified the identified silkworm BTB protein genes into nine subfamilies: BTB only, BTB-ANK, BTB-BACK, BTBBACK-PHR, BTB-BACK-Kelch, BTB-Kelch, BTB-ZF, BTB$\mathrm{HTH}$, and MATH-BTB. The presence of various additional functional domains in the silkworm BTB protein genes suggests that the BTB protein genes in different subfamilies may have diverse functions.

Our comparative analysis revealed that BTB protein genes have been conserved to a certain extent during insect evolution. This conclusion is supported by the following evidence. First, the total number of BTB protein genes present in the silkworm genome and in the genomes of four other surveyed insect species is similar, indicating that no expansion has occurred in the BTB protein gene family during insect evolution. This finding differs from a previous observation that BTB protein genes have undergone a lineage-specific expansion in vertebrates in a comparison with the fruit fly and the malaria mosquito [6]. Second, we noted that the members of some BTB protein gene subfamilies showed a rigorous orthologous relationship among the silkworm and other insects, suggesting that these members may have similar functions in the surveyed insect species. For example, the orthologs of BR-C (ZBTB6) or fruitless (ZBTB5), two well-studied insect BTB protein genes, were grouped into a clade on the phylogenetic tree (Figure 4). This finding 

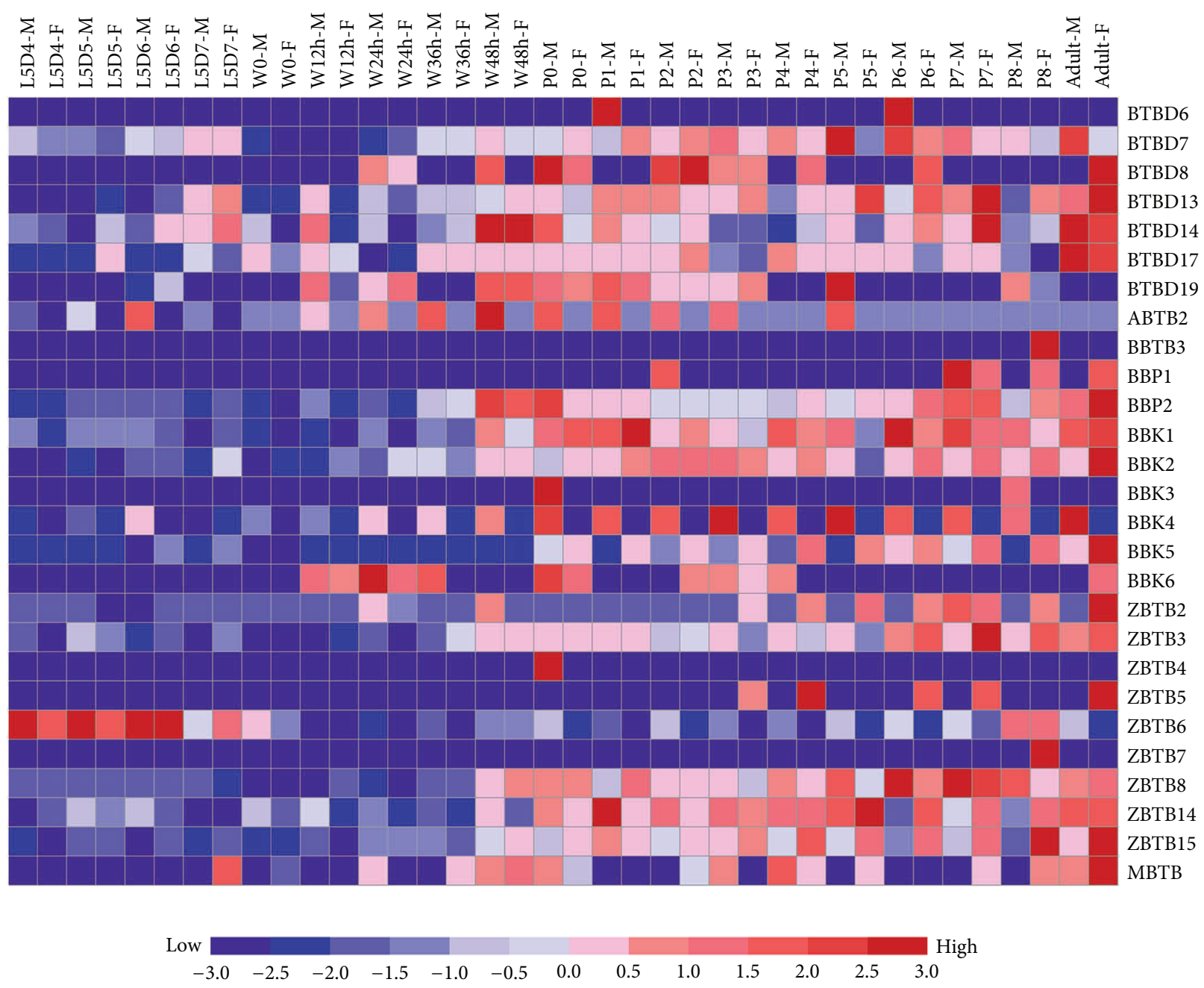

FIGURE 7: Microarray-based expression profiling of the BTB protein genes during silkworm metamorphosis. Time-course microarray data representing gene expression during silkwormmetamorphosis was used to profile the developmental expression patterns of silkworm BTB protein genes. Changes in the expression of the BTB protein genes were visualized using the GeneCluster 2.0 program. Plus and minus denote up- and downregulation, respectively. F: female; M: male; L: larval; W: wandering; P: pupal; D: day; h: hour. The Arabic numeral in phase P1 represents one day after pupation.

is consistent with the known conserved functions of BR-C protein in ecdysone signaling [7] and with the contribution of fruitless protein to the regulation of male sexual behavior and sexual orientation [43]. In addition, previous studies have shown that MATH-BTB proteins are ubiquitously present in plants and metazoa [6] and they mainly function as substratebinding adapters for the Cullin3-based ubiquitin E3 ligase that is involved in the ubiquitination and proteolysis of target proteins [44-46]. Because ubiquitination-induced protein degradation also occurs in the fruit fly $[47,48]$, we reasoned that MATH-BTB protein-mediated ubiquitination should occur widely in insects. Third, in all insect species examined, the BTB-BACK-Kelch subfamily possesses a similar number of members and has experienced a moderate expansion when comparing to this subfamily in yeast [6]. This observation, coupled with the finding that the BTB-BACK-Kelch subfamilies in the surveyed insects were grouped together in the phylogenetic tree of all insect BTB protein genes but form different subclades based on sequence similarity and not species identity (Figures 3 and 4 ), suggests that the expansion of the BTB-BACK-Kelch subfamily has occurred before insect radiation.

Our work also shows that evolutionary divergence has also occurred among insect BTB protein genes. First, in agreement with previous observations [6], the number of BTB protein genes in the silkworm and other insect species is apparently smaller than that in mammals and larger than that in yeast, further confirming the occurrence of lineage-specific expansions of BTB protein genes. Second, different members of the BTB only subfamily were always separately grouped with the members of other subfamilies in the phylogenetic trees constructed either from silkworm BTB protein genes or from all insect BTB protein genes, suggesting that the $\mathrm{BTB}$ protein genes that contain additional domains may have evolved from these BTB protein genes of the BTB only subfamily. Similarly, some members of 
the BTB-ZF subfamily were also grouped together with the members of BTB only proteins before grouping with other members of BTB-ZF subfamily in the phylogenetic tree based on the complete amino acid sequences of silkworm BTB proteins, indicating that these BTB-ZF proteins may play distinct roles. Third, we noted that the BTB-BACK-PHR and RhoBTB subfamilies were not present in some insect species. Whether this lack resulted from the genome gap or whether these subfamilies actually play specific roles in the insect species in which they are present remains to be determined.

Another important finding of our study is that several silkworm BTB protein genes exhibited spatiotemporally specific expression profiles. Interestingly, silkworm $A B T B 2$, $B B K 3$, and $B B K 4$ were expressed specifically in the testis of mid-fifth instar larvae and in male individuals during metamorphosis. The silkworm ABTB2 gene encodes an inhibitor of Bruton tyrosine kinase (BTK), which has been shown to be involved in male genital development and survival in the fruit fly $[49,50]$. Therefore, the male-specific expression of the silkworm $A B T B 2$ gene suggests that this gene may be involved in the regulation of male genital development in the silkworm. In addition, we noted that ZBTB5 gene showed no sex-dependent expression on L5D3 but was specifically expressed in the female silkworm during the pupa-adult transition. Given that during oogenesis the growth and meiosis of the oocytes and egg formation occur during the pupa-adult transition of the silkworm [51], we speculate that ZBTB5 may play key roles in the regulation of silkworm oogenesis. Future studies are necessary to determine how sex-specific expression of these BTB protein genes functions during silkworm development.

\section{Abbreviations}

BTB: Bric-a-brac, tramtrack, and broad complex

ZF: $\quad$ Zinc finger

BACK: BTB and C-terminal Kelch

MATH: Meprin and TRAF homology

ANK: Ankyrin

Rho: Ras homology

BR-C: Broad complex.

\section{Conflict of Interests}

The authors declare that there is no conflict of interests regarding the publication of this paper.

\section{Authors' Contribution}

Daojun Cheng and Wenliang Qian contributed equally to this work.

\section{Acknowledgments}

This work was supported by Grants from the National Natural Science Foundation of China (no. 31172267 and no. 31272503), the National Basic Research Program of China (no. 2012CB114600), the National Hi-Tech Research and Development Program of China (no. 2011AA100306), and the Municipal Natural Science Foundation of Chongqing (no. cstc2012jjA80023).

\section{References}

[1] S. Zollman, D. Godt, G. G. Privé, J. Couderc, and F. A. Laski, "The BTB domain, found primarily in zinc finger proteins, defines an evolutionarily conserved family that includes several developmentally regulated genes in Drosophila," Proceedings of the National Academy of Sciences of the United States of America, vol. 91, no. 22, pp. 10717-10721, 1994.

[2] V. J. Bardwell and R. Treisman, "The POZ domain: a conserved protein-protein interaction motif," Genes and Development, vol. 8, no. 14, pp. 1664-1677, 1994.

[3] T. Collins, J. R. Stone, and A. J. Williams, "All in the family: the BTB/POZ, KRAB, and SCAN domains," Molecular and Cellular Biology, vol. 21, no. 11, pp. 3609-3615, 2001.

[4] M. Numoto, O. Niwa, J. Kaplan et al., "Transcriptional repressor ZF5 identifies a new conserved domain in zinc finger proteins," Nucleic Acids Research, vol. 21, no. 16, pp. 3767-3775, 1993.

[5] R. Perez-Torrado, D. Yamada, and P. A. Defossez, "Born to bind: the BTB protein-protein interaction domain," BioEssays, vol. 28, no. 12, pp. 1194-1202, 2006.

[6] P. J. Stogios, G. S. Downs, J. J. S. Jauhal, S. K. Nandra, and G. G. Privé, "Sequence and structural analysis of BTB domain proteins," Genome Biology, vol. 6, no. 10, article R82, 2005.

[7] F. D. Karim, G. M. Guild, and C. S. Thummel, “The Drosophila Broad-Complex plays a key role in controlling ecdysoneregulated gene expression at the onset of metamorphosis," Development, vol. 118, no. 3, pp. 977-988, 1993.

[8] G. Tzolovsky, W. Deng, T. Schlitt, and M. Bownes, "The function of the Broad-Complex during Drosophila melanogaster oogenesis," Genetics, vol. 153, no. 3, pp. 1371-1383, 1999.

[9] W. Li, F. Wang, L. Menut, and F. B. Gao, "BTB/POZ-zinc finger protein abrupt suppresses dendritic branching in a neuronal subtype-specific and dosage-dependent manner," Neuron, vol. 43, no. 6, pp. 823-834, 2004.

[10] Y. Wen, D. Nguyen, Y. Li, and Z. C. Lai, "The N-terminal $\mathrm{BTB} / \mathrm{POZ}$ domain and C-terminal sequences are essential for Tramtrack69 to specify cell fate in the developing Drosophila eye," Genetics, vol. 156, no. 1, pp. 195-203, 2000.

[11] S. L. Haigo, R. M. Harland, and J. B. Wallingford, "A family of Xenopus BTB-Kelch repeat proteins related to ENC-1: new markers for early events in floorplate and placode development," Gene Expression Patterns, vol. 3, no. 5, pp. 669-674, 2003.

[12] L. Korutla, J. H. Neustadter, K. M. Fournier, and S. A. Mackler, "NAC1, a POZ/BTB protein present in the adult mammalian brain, triggers apoptosis after adenovirus-mediated overexpression in PC-12 cells," Neuroscience Research, vol. 46, no. 1, pp. 3339, 2003.

[13] K. Sasagawa, Y. Matsudo, M. Kang et al., "Identification of Nd1, a novel murine kelch family protein, involved in stabilization of actin filaments," The Journal of Biological Chemistry, vol. 277, no. 46, pp. 44140-44146, 2002.

[14] S. Fujiyama-Nakamura, S. Ito, S. Sawatsubashi et al., "BTB protein, dKLHL18/CG3571, serves as an adaptor subunit for a dCul3 ubiquitin ligase complex," Genes to Cells, vol. 14, no. 8, pp. 965-973, 2009. 
[15] P. Rondou, G. Haegeman, P. Vanhoenacker, and K. van Craenenbroeck, "BTB protein KLHL12 targets the dopamine D4 receptor for ubiquitination by a Cul3-based E3 ligase," The Journal of Biological Chemistry, vol. 283, no. 17, pp. 11083-11096, 2008.

[16] P. Figueroa, G. Gusmaroli, G. Serino et al., "Arabidopsis has two redundant cullin3 proteins that are essential for embryo development and that interact with RBX1 and BTB proteins to form multisubunit E3 ubiquitin ligase complexes in vivo," The Plant Cell, vol. 17, no. 4, pp. 1180-1195, 2005.

[17] M. Furukawa and Y. Xiong, "BTB protein keap1 targets antioxidant transcription factor Nrf2 for ubiquitination by the cullin 3-Roc1 ligase," Molecular and Cellular Biology, vol. 25, no. 1, pp. 162-171, 2005.

[18] S. Luke-Glaser, L. Pintard, C. Lu, P. E. Mains, and M. Peter, "The BTB protein MEL-26 promotes cytokinesis in C. elegans by a CUL-3-independent mechanism," Current Biology, vol. 15, no. 18, pp. 1605-1615, 2005.

[19] T. G. Nacak, K. Leptien, D. Fellner, H. G. Augustin, and J. Kroll, "The BTB-kelch protein LZTR-1 is a novel Golgi protein that is degraded upon induction of apoptosis," The Journal of Biological Chemistry, vol. 281, no. 8, pp. 5065-5071, 2006.

[20] J. Berthold, K. Schenková, and F. Rivero, "Rho GTPases of the RhoBTB subfamily and tumorigenesis," Acta Pharmacologica Sinica, vol. 29, no. 3, pp. 285-295, 2008.

[21] X. Q. Liang, H. K. Avraham, S. Jiang, and S. Avraham, "Genetic alterations of the NRP/B gene are associated with human brain tumors," Oncogene, vol. 23, no. 35, pp. 5890-5900, 2004.

[22] A. Melnick, G. Carlile, K. F. Ahmad et al., "Critical residues within the BTB domain of PLZF and Bcl- 6 modulate interaction with corepressors," Molecular and Cellular Biology, vol. 22, no. 6, pp. 1804-1818, 2002.

[23] A. Bonchuk, S. Denisov, P. Georgiev, and O. Maksimenko, "Drosophila BTB/POZ domains of "ttk group" can form multimers and selectively interact with each other," Journal of Molecular Biology, vol. 412, no. 3, pp. 423-436, 2011.

[24] H. Weber, A. Bernhardt, M. Dieterle et al., "Arabidopsis AtCUL3a and AtCUL3b form complexes with members of the BTB/POZ-MATH protein family," Plant Physiology, vol. 137, no. 1, pp. 83-93, 2005.

[25] J. H. Cho, M. J. Kim, K. J. Kim, and J. R. Kim, "POZ/BTB and AT-hook-containing zinc finger protein 1 (PATZ1) inhibits endothelial cell senescence through a p53 dependent pathway," Cell Death and Differentiation, vol. 19, no. 4, pp. 703-712, 2012.

[26] Q. Liu, F. Yao, M. Wang et al., "Novel human BTB/POZ domaincontaining zinc finger protein ZBTB1 inhibits transcriptional activities of CRE," Molecular and Cellular Biochemistry, vol. 357, no. 1-2, pp. 405-414, 2011.

[27] J. Qi, X. Zhang, H. K. Zhang, H. M. Yang, Y. B. Zhou, and Z. G. Han, "ZBTB34, a novel human BTB/POZ zinc finger protein, is a potential transcriptional repressor," Molecular and Cellular Biochemistry, vol. 290, no. 1-2, pp. 159-167, 2006.

[28] L. Korutla, P. J. Wang, and S. A. Mackler, "The POZ/BTB protein NAC1 interacts with two different histone deacetylases in neuronal-like cultures," Journal of Neurochemistry, vol. 94, no. 3, pp. 786-793, 2005.

[29] T. Ijiro, H. Urakawa, Y. Yasukochi, M. Takeda, and Y. Fujiwara, "cDNA cloning, gene structure, and expression of BroadComplex (BR-C) genes in the silkworm, Bombyx mori," Insect Biochemistry and Molecular Biology, vol. 34, no. 9, pp. 963-969, 2004.
[30] M. Uhlirova, B. D. Foy, B. J. Beaty, K. E. Olson, L. M. Riddiford, and M. Jindra, "Use of Sindbis virus-mediated RNA interference to demonstrate a conserved role of Broad-Complex in insect metamorphosis," Proceedings of the National Academy of Sciences of the United States of America, vol. 100, no. 26, pp. 15607-15612, 2003.

[31] The International Silkworm Genome Consortium, "The genome of a lepidopteran model insect, the silkworm Bombyx mori," Insect Biochemistry and Molecular Biology, vol. 38, no. 12, pp. 1036-1045, 2008.

[32] C. P. Ponting, J. Schultz, F. Milpetz, and P. Bork, "SMART: identification and annotation of domains from signalling and extracellular protein sequences," Nucleic Acids Research, vol. 27, no. 1, pp. 229-232, 1999.

[33] A. Marchler-Bauer, S. Lu, J. B. Anderson et al., "CDD: a conserved domain database for the functional annotation of proteins," Nucleic Acids Research, vol. 39, no. 1, pp. D225-D229, 2011.

[34] K. Yamamoto, J. Nohata, K. Kadono-Okuda et al., "A BAC-based integrated linkage map of the silkworm Bombyx mori," Genome Biology, vol. 9, no. 1, article R21, 2008.

[35] P. Horton, K. Park, T. Obayashi et al., "WoLF PSORT: protein localization predictor," Nucleic Acids Research, vol. 35, pp. W585-W587, 2007.

[36] M. A. Larkin, G. Blackshields, N. P. Brown et al., "Clustal W and clustal X version 2.0," Bioinformatics, vol. 23, no. 21, pp. 29472948, 2007.

[37] K. Tamura, J. Dudley, M. Nei, and S. Kumar, "MEGA4: molecular evolutionary genetics analysis (MEGA) software version 4.0," Molecular Biology and Evolution, vol. 24, no. 8, pp. 15961599, 2007.

[38] Q. Xia, D. Cheng, J. Duan et al., "Microarray-based gene expression profiles in multiple tissues of the domesticated silkworm, Bombyx mori," Genome Biology, vol. 8, no. 8, article R162, 2007.

[39] M. Reich, K. Ohm, M. Angelo, P. Tamayo, and J. P. Mesirov, "GeneCluster 2.0: an advanced toolset for bioarray analysis," Bioinformatics, vol. 20, no. 11, pp. 1797-1798, 2004.

[40] D. Cheng, Q. Xia, J. Duan et al., "Nuclear receptors in Bombyx mori: insights into genomic structure and developmental expression," Insect Biochemistry and Molecular Biology, vol. 38, no. 12, pp. 1130-1137, 2008.

[41] O. Albagli, P. Dhordain, C. Deweindt, G. Lecocq, and D. Leprince, "The BTB/POZ domain: a new protein-protein interaction motif common to DNA- and actin-binding proteins," Cell Growth and Differentiation, vol. 6, no. 9, pp. 1193-1198, 1995.

[42] K. F. Ahmad, C. K. Engel, and G. G. Privé, "Crystal structure of the BTB domain from PLZF", Proceedings of the National Academy of Sciences of the United States of America, vol. 95, no. 21, pp. 12123-12128, 1998.

[43] L. C. Ryner, S. F. Goodwin, D. H. Castrillon et al., "Control of male sexual behavior and sexual orientation in Drosophila by the fruitless gene," Cell, vol. 87, no. 6, pp. 1079-1089, 1996.

[44] J. H. Thomas, "Adaptive evolution in two large families of ubiquitin-ligase adapters in nematodes and plants," Genome Research, vol. 16, no. 8, pp. 1017-1030, 2006.

[45] M. Juranic, K. O. Srilunchang, N. G. Krohn, D. Leljak-Levanic, S. Sprunck, and T. Dresselhaus, "Germline-specific MATHBTB substrate adaptor MAB1 regulates spindle length and nuclei identity in maize," The Plant Cell, vol. 24, no. 12, pp. 49744991, 2012. 
[46] M. Zhuang, M. F. Calabrese, J. Liu et al., "Structures of SPOPsubstrate complexes: insights into molecular architectures of BTB-Cul3 ubiquitin ligases," Molecular Cell, vol. 36, no. 1, pp. 39-50, 2009.

[47] C. Y. Ou, H. Pi, and C. T. Chien, "Control of protein degradation by E3 ubiquitin ligases in Drosophila eye development," Trends in Genetics, vol. 19, no. 7, pp. 382-389, 2003.

[48] A. A. Freeman, K. Mandilaras, F. Missirlis, and S. Sanyal, "An emerging role for Cullin-3 mediated ubiquitination in sleep and circadian rhythm: insights from Drosophila," Fly, vol. 7, no. 1, pp. 39-43, 2013.

[49] N. Hamada, C. Bäckesjö, C. I. E. Smith, and D. Yamamoto, "Functional replacement of Drosophila Btk29A with human Btk in male genital development and survival," FEBS Letters, vol. 579, no. 19, pp. 4131-4137, 2005.

[50] K. Baba, A. Takeshita, K. Majima et al., “The Drosophila Bruton's tyrosine kinase (Btk) homolog is required for adult survival and male genital formation," Molecular and Cellular Biology, vol. 19, no. 6, pp. 4405-4413, 1999.

[51] Z. Xiang, Study of Silkworm Egg Production in China, Sichuan Scientific and Technical Press, 1995 (Chinese). 

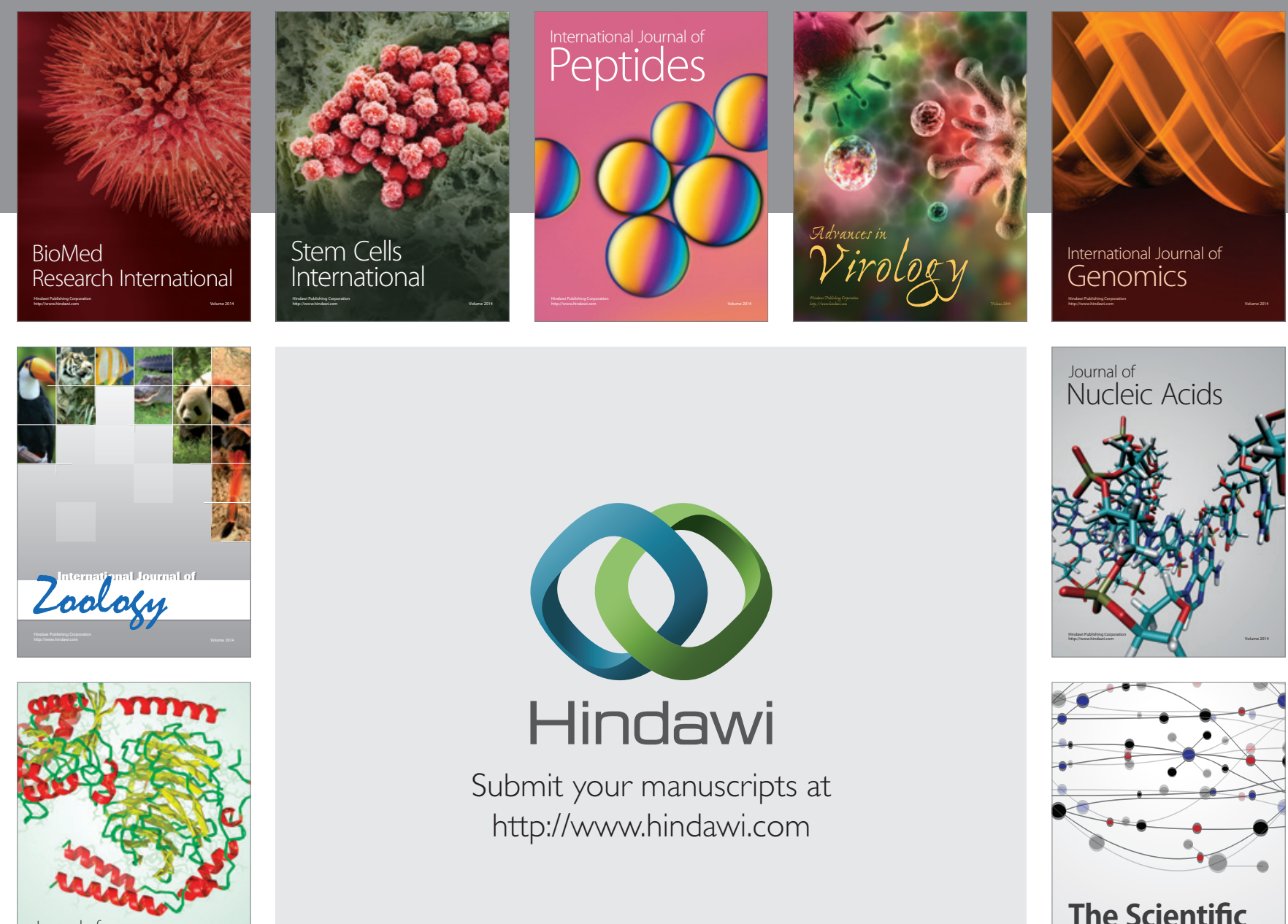

Submit your manuscripts at

http://www.hindawi.com

Journal of
Signal Transduction
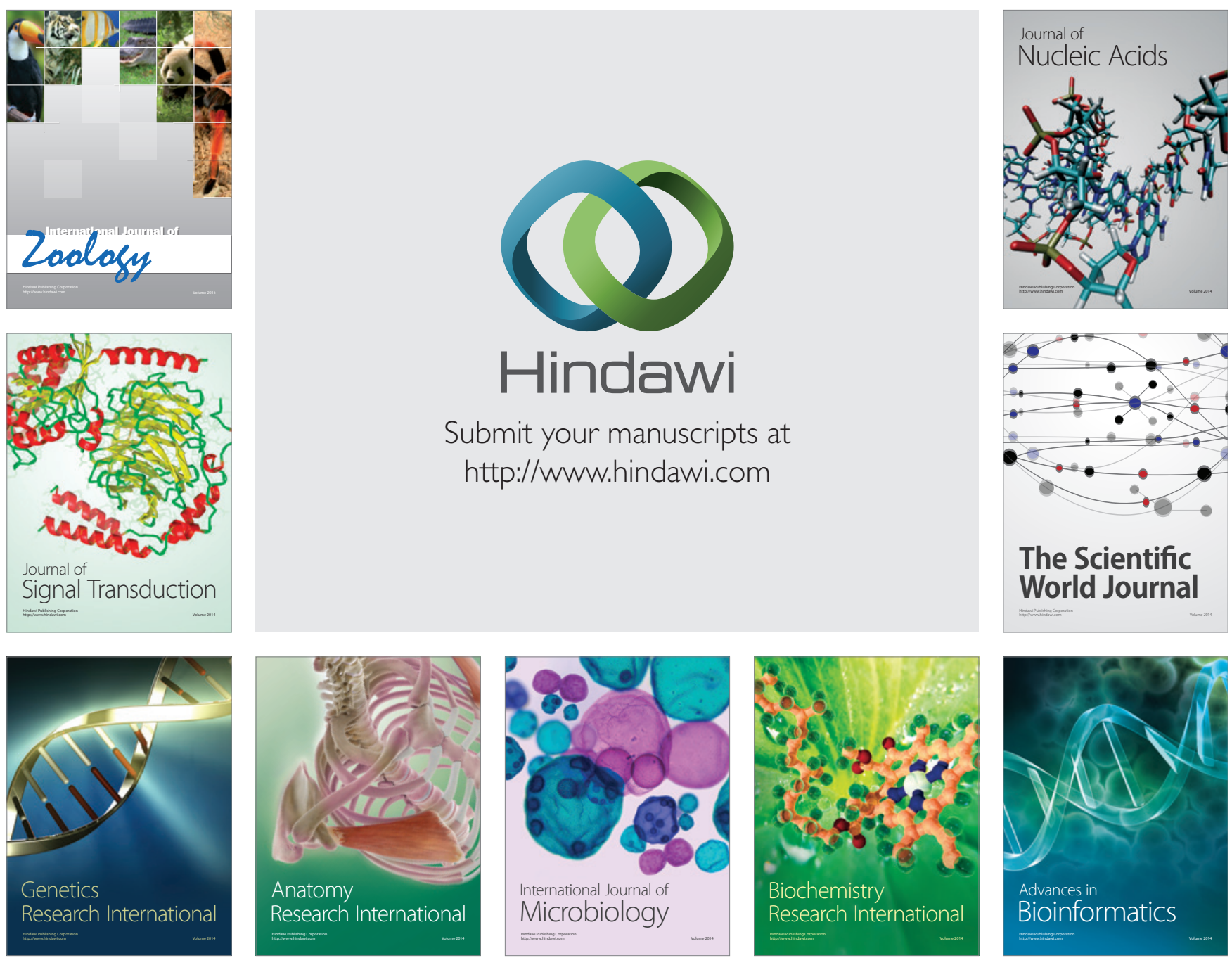

The Scientific World Journal
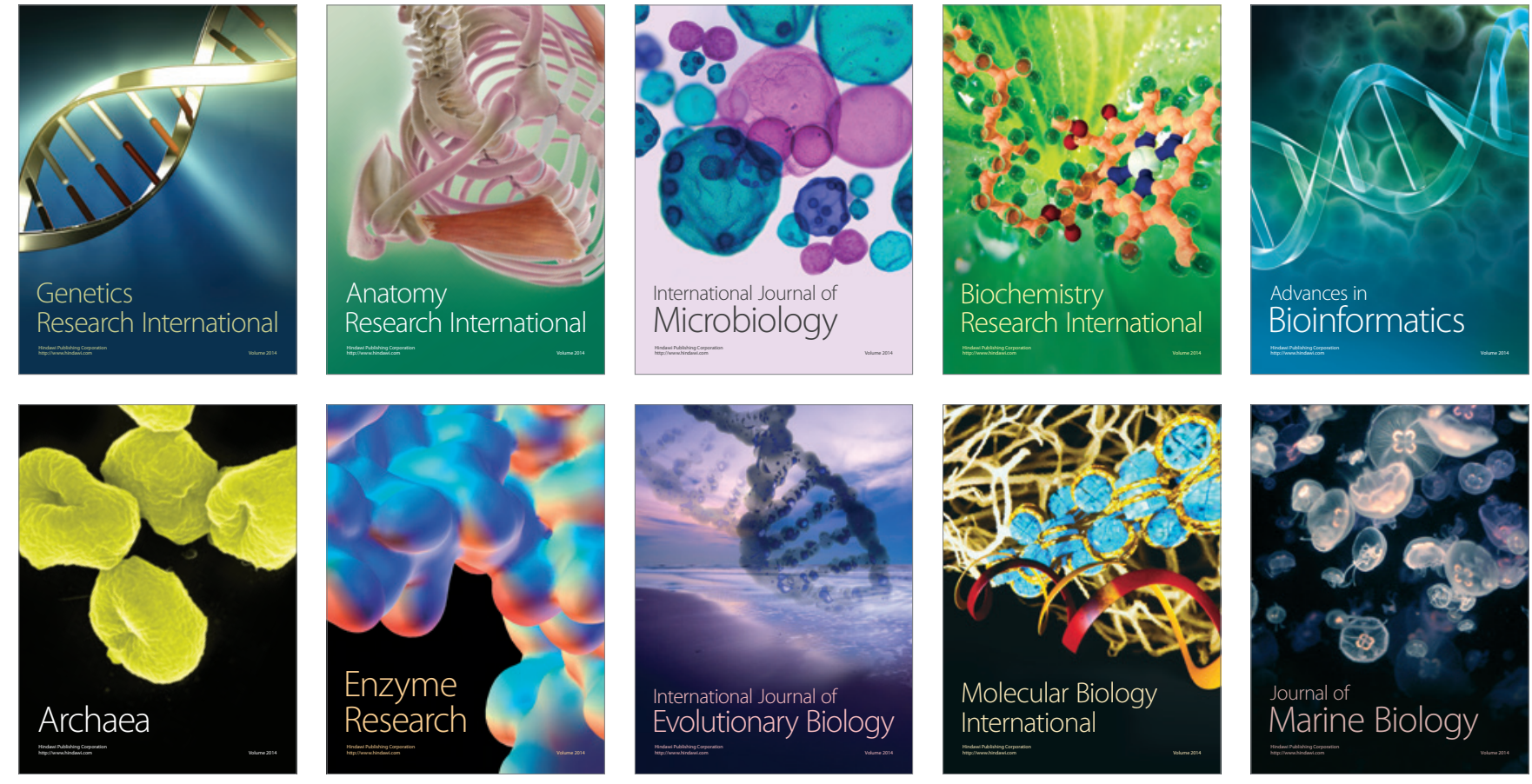